\title{
ALGORITMO GERAL PARA A PROCURA DE CÓDIGOS TRELIÇA ÓTIMOS BASEADOS EM PARTIÇÕES DE RETICULADOS
}

\author{
E. M. Rosa*, W. C. Borelli ${ }^{\dagger}$ e P. G. Farrell ${ }^{\ddagger}$ \\ *Departamento de Matemática - Fac. de Ciências - Unesp - Bauru - SP, emilia@ bauru.unesp.br \\ tDepartamento de Telemática - FEEC - Unicamp - Campinas - SP, borelli@ dt.unicamp.br \\ ‡Departamento de Eng. Elétrica, Univ. of Manchester - U.K., farrell@man.ac.uk
}

Resumo - Neste artigo é proposto um algoritmo geral de procura de códigos treliça ótimos q-ários, baseados em reticulados e suas partiçōes, o qual incorpora os procedimentos introduzidos para códigos binários em [ [2],[4], e generaliza a procura de códigos ótimos para alfabetos que são anéis com q elementos, $Z_{q}$. Novas características foram incorporadas ao algoritmo, proporcionando uma procura de códigos ótimos mais rápida e eficiente do que em [ [3], [4],[5]]. Exemplos numéricos de aplicação do algoritmo para um esquema binário com a partiçäo $Z^{2} / \theta Z^{2}$ e para um esquema ternário com a partiçằo $A_{2} / 9 A_{2}$, são apresentados em detalhes.

Abstract - In this paper a general algorithm for the search of optimum q-ary trellis codes, based on lattices and cosets, is proposed. The algorithm incorporates the procedures introduced for binary codes in [.[2],[4]] and generalizes the search for alphabets that are rings with q elements, $Z_{q}$. New characteristics were incorporated to the algorithm, providing a faster and more efficient search for the optimum codes than in [.[3], [4],[5]]. Numerical examples are presented in details for binary scheme with the $Z^{2} / \theta Z^{2}$ partition and for a temary scheme with the $A_{2} / 9 A_{2}$ partition.

Palavras-chave : códigos treliça, códigos convolucionais, partiçōes de reticulados, cosets, códigos ótimos, algoritmo de procura.

\section{INTRODUÇÃO}

Seja a estrutura geral do codificador de um código treliça, como em .1 e que é mostrado na fig, o qual transforma uma sequência de símbolos de um alfabeto $\mathcal{A}$ em uma sequência de símbolos de saída, os quais mapeiam uma constelação num reticulado $\Lambda$ do $\mathcal{R}^{n}$.

Considere $\mathcal{A}$ um anel finito, $\Lambda^{\prime} \subseteq \Lambda$ um subreticulado, $\mathrm{G}$ a matriz geradora de um código convolucional com $k_{1}$ símbolos de entrada de $\mathcal{A}$ e apenas um símbolo de saída, $\mathrm{r}$, correspondente a um coset $\left(\mathrm{g}_{i j}\right)$ da partição de reticulados $\Lambda / \Lambda^{\prime}$.

O codificador do código treliça, possui $k=k_{1}+k_{2}$ bits de entrada e um ponto da constelação como saída, sendo $k_{1}$, os bits codificados pelo código convolucional e $k_{2}$, os símbolos de entrada não codificados, os quais são utilizados na escolha de um ponto específico dentre os $\alpha^{k_{2}}$ pontos do coset, previamente escolhido pelo codificador convolucional.

A taxa do código convolucional é $k_{1} \log _{2} \alpha$ bits/coset e a taxa fracionária e $\rho=k_{1} \log _{2} \alpha / \log _{2}|T|$ onde $\alpha=|A|$

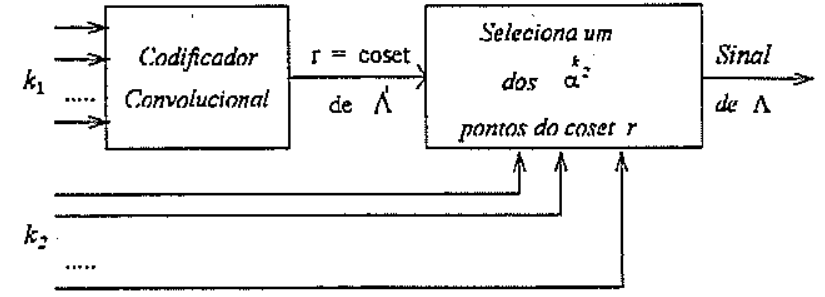

FIG. 1: Estrutura do Codificador de um Código Treliça[1].

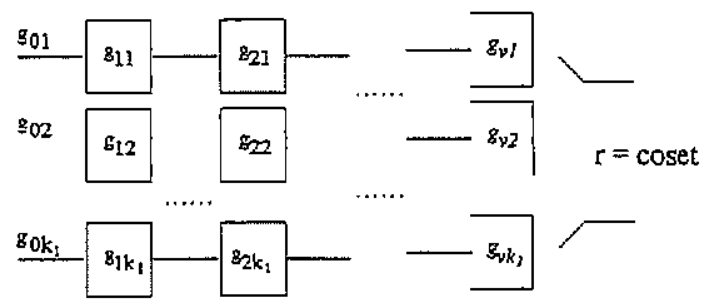

FIG. 2: Estrutura do Código Convolucional.

e $|T|$ e o número de saídas possíveis sendo $\alpha^{k_{1}}$ o número de entradas possíveis. O codificador pode determinar até $\alpha^{k_{1}+V}$ cosets distintos.

A fig.2 apresenta a estrutura genérica do codificador convolucional com $k_{1}$ entradas e $v=\max _{1 \leq j \leq k_{1}}\left\{v_{j}\right\} ; v_{j}$ é o número de memórias em cada entrada do codificador, onde $\mathrm{g}_{\mathrm{vj}}=0$, sempre que $v_{j}<v$.

Denomina-se 1 matriz geradora do código convolucional envolvido na estrutura do Código Treliça, a matriz $\mathrm{G}$ que tem cosets $\mathrm{g}_{\mathfrak{i j}}$ como suas colunas :

$$
G=\left[g_{v k_{1}} \ldots g_{v 1}|\ldots| g_{1 k_{1}} \ldots g_{11} \mid g_{0 k_{1}} \ldots g_{01}\right]
$$

Define-se como saída do codificador convolucional, o coset

$$
r=\sum_{i=0}^{v} \sum_{j=1}^{k_{1}} u_{i j} \mathrm{~g}_{i j}
$$

onde $u_{i j} \in \mathcal{A}$ e $\mathrm{g}_{\mathrm{ij}} \in \Lambda / \Lambda^{\prime}$.

A norma de umo coset $e$ definida como $\left\|g_{i j}\right\|=\sum_{k} x_{i j_{k}}^{2}$, onde $k=1,2, \ldots, \operatorname{dim}(\Lambda) ; x_{i j_{k}}$ são as coordenadas Euclidianas do coset $g_{i j}$ no reticulado $\Lambda$.1.

A distância mínima de um código treliça é definida em .1 pela expressão

$$
d=\min \left\{d_{f r e e}, d_{2}\right\}
$$




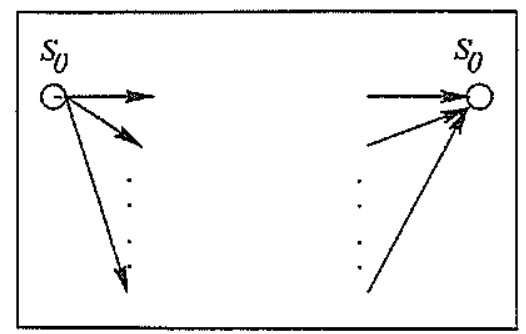

FIG. 3: Limitante inferior para o $d_{\text {free }}$.

sendo $d_{\text {free }}$ o valor mínimo dentre as métricas dos caminhos fechados com início, e final, no estado zero da treliça e $d_{2}$, a distância Euclidiana quadrada mínima, obtida entre os pontos de um mesmo coset da partição de reticulados. Dados os va- lores de $k_{1}, k_{2}, \mathrm{~V}$, q, e uma partição especifica de reticulados, defini-se código treliça ótimo como sendo o que possui o valor máximo para a distância mínima d. A maximização da distância mínima d, necessariamente, passa pela maximização do parâmetro $d_{f r e e}$ do código convolucional, pois $d_{2}$ tem valor fixado pela partição.

$O$ aumento do número de memórias de um codificador convolucional pode proporcionar um valor maior para o $d_{\text {free }}$ do código gerado. Desta forma os parâmetros, $\left(k_{1}, \mathrm{~V}, \mathrm{q}\right)$ e a partição, $\Lambda / \Lambda^{\prime}$, são considerados de modo que os esquemas gerem códigos convolucionais onde $d_{\text {free }} \leq d_{2}$.

Este artigo propõe um algoritmo geral de procura de códigos convolucionais ótimos q-ários, o qual é apresentado na seção III, de maneira bastante detalhada O algoritmo incorpora e generaliza os procedimentos apresentados em [.[2],[2]], visto que se procura códigos convolucionais não catastróficos com $d_{\text {free }}$ máximo, i.e., códigos ótimos, binários .3 e q-ários [.[2],[5], [6]] para um dado conjunto de parâmetros $\left(k_{1}, \mathrm{~V}, \mathrm{q}\right)$ e uma dada partição de reticulados $\Lambda / \Lambda^{\prime}$.

\section{SUBCONJUNTOS ESPECIAIS DE MATRIZES GERADORAS}

Seja $\Delta_{i n f}$, um limitante inferior para o $d_{\text {free }}$ de um código convolucional, formado pela soma da menor métrica dentre os ramos que chegam ao estado zero, $\left(S_{0}\right)$, com a menor métrica dentre os ramos que partem do referido estado.

Por outro lado a métrica de um caminho fechado qualquer da treliça limita superiormente $0 d_{\text {free }}$ do código convolucional. Portanto considere o parâmetro, $\Delta_{\text {sup }}$, como sendo a menor métrica obtida a partir de um conjunto finito de caminhos fechados da treliça (caminhos com início e fim no estado $S_{0}$ ), como mostrado na fig. 4 .

A determinação das matrizes geradoras de bons códigos convolucionais (códigos não catastróficos com $d_{f r e e}$ grande), envolve uma escolha adequada de suas colunas, a qual deve propiciar a maximização dos parâmetros $\Delta_{i n f}$ e $\Delta_{s u p}$, bem como, possibilitar a obtenção de todos os cosets da partição utilizada, na saída đo codificador convolucional. A partir desta escolha obtem-se subconjuntos especiais .4 com um número reduzido de matrizes geradoras, dentre os quais existem matrizes que geram códigos ótimos ( $d_{f r e e}$ máximo).

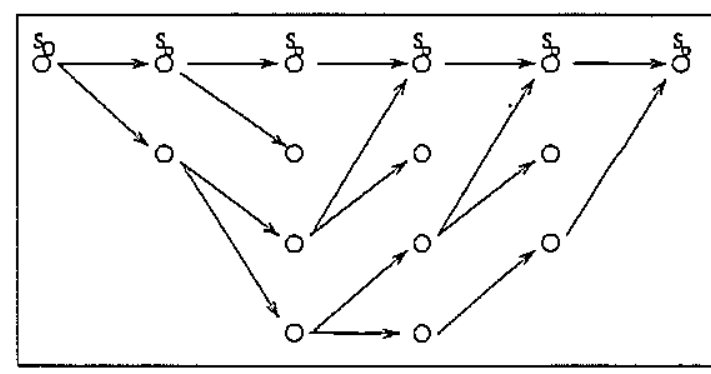

FIG. 4: Limitante superior para $d_{\text {free }}$.

Cada subconjunto especial de matrizes geradoras $G(1)$, pode ser representado por uma matriz-norma GN .7

$$
G N=\left[\left\|\mathbf{g}_{\mathbf{v k}}\right\| \ldots\left\|\mathbf{g}_{\mathbf{v} 1}\right\||\ldots|\left\|\mathbf{g}_{0 \mathbf{k}_{1}}\right\| \ldots\left\|\mathbf{g}_{01}\right\|\right]
$$

na qual, as colunas são escolhidas dentre as normas dos cosets da partição, de forma que maximizem os limitantes, $\Delta_{\text {inf }} \mathrm{e}$ $\Delta_{\text {sup }}$, do $d_{\text {free }}$ dos códigos gerados pelas matrizes $G$ pertencentes ao subconjunto especial.

\subsection{Definições do $\Delta_{i n f}$ e $\Delta_{\text {sup }}$ dos Subconjun- tos Especiais}

Dado o conjunto de blocos de bits de entrada :

$$
\mathcal{U}=\left\{\begin{array}{c}
\left\{\mathbf{u}_{1}\right\}=\left(u_{l_{k_{1}}}, \ldots, u_{l_{1}}\right) \mid\left\{\mathbf{u}_{1}\right\} \neq\{0\}, \\
\left\{\mathbf{u}_{1}\right\} \in \mathcal{A}^{k_{2}}, l=1, \ldots, \alpha^{k_{1}}
\end{array}\right\},
$$

As definiçōes 4, dos parâmetros $\Delta_{i n f}$ e $\Delta_{s u p}$, podem ser generalizadas para quaisquer valores de $k_{1} \mathrm{e} V$ pelas seguintes expressōes :

$$
\Delta_{i n f}=\min _{\left\{\mathbf{u}_{l}\right\}}\left\{\left\|\sum_{j=1}^{k_{1}} u_{l_{j}} \mathbf{g}_{\mathbf{v} j}\right\|\right\}+\min _{\left\{\mathbf{u}_{l}\right\}}\left\{\left\|\sum_{j=1}^{k_{1}} u_{l_{j}} \mathbf{g}_{0_{j}}\right\|\right\}
$$

onde os cosets $\mathrm{g}_{0 \mathrm{j}}$ estão associados aos ramos que partem do estado $S_{0}$ e os $\mathrm{g}_{\mathrm{vj}}$, aos que chegam a esse estado da treliça conforme fig. $3 \mathrm{e}$

$$
\Delta_{\text {sup }}=\min _{\left\{\mathbf{u}_{1}\right\}}\left\{\sum_{i=0}^{v}\left\|\sum_{j=1}^{k_{1}} u_{l_{j}} \mathrm{~g}_{\mathfrak{i j}}\right\|\right\}
$$

sendo que dentre todas as sequências de símbolos de entrada, nesta expressão utilizou-se somente aquelas associadas aos caminhos fechados cujo número de ramos na treliça cor- respondente é mínimo.

Em geral pode-se considerar mais caminhos fechados da treliça para a definição do $\Delta_{s u p}$ o que poderá proporcionar maior rapidez na obtenção de um código ótimo [.[5]. [6]].

Supondo o caso de $k_{1}=2 \mathrm{e} \mathcal{A}=G F(2)$, as expressōes (4), (5) e (6) ficariam reduzidas a:

$$
\begin{gathered}
\mathcal{U}=\{\{0,1\},\{1,0\},\{1,1\}\} ; \\
\Delta_{i n f}=\min \left\{\begin{array}{l}
\left\|0 . g_{v 1}+1 . g_{v 2}\right\|, \\
\left\|1 . g_{v 1}+0 . g_{v 2}\right\|, \\
\left\|1 . g_{v 1}+1 . g_{v 2}\right\|
\end{array}\right\}+
\end{gathered}
$$




$$
\begin{gathered}
+\min \left\{\begin{array}{l}
\left\|0 . g_{01}+1 . g_{02}\right\|, \\
\left\|1 . g_{01}+0 . g_{02}\right\|, \\
\left\|1 . g_{01}+1 . g_{02}\right\|
\end{array}\right\} \\
=\min \left\{\begin{array}{l}
\left\|g_{v 2}\right\|, \\
\left\|g_{v 1}\right\|, \\
\left\|g_{v 1}+g_{v 2}\right\|
\end{array}\right\}+\min \left\{\begin{array}{l}
\left\|g_{02}\right\|, \\
\left\|g_{01}\right\|, \\
\left\|g_{01}+g_{02}\right\|
\end{array}\right\} ; \\
\Delta_{\text {sup }}=\min \left\{\begin{array}{l}
\sum_{i=0}^{v}\left\|0 . g_{i 1}+1 . g_{i 2}\right\|, \\
\sum_{i=0}^{v}\left\|1 . g_{i 1}+0 . g_{i 2}\right\|, \\
\sum_{i=0}\left\|1 . g_{i 1}+1 . g_{i 2}\right\|
\end{array}\right\} \\
=\min \left\{\sum_{i=0}^{v}\left\|g_{i 2}\right\|, \sum_{i=0}^{v}\left\|g_{i 1}\right\|, \sum_{i=0}^{v}\left\|g_{i 1}+g_{i 2}\right\|\right\} .
\end{gathered}
$$

\subsection{Procedimentos para uma escolha eficaz dos subconjuntos especiais}

a) Dada uma partição de reticulados, calcule a expressão :

$$
\max _{\mathbf{g}_{\mathbf{j}} \in \Lambda / \Lambda^{\prime}}\left\{\min _{\left\{\mathbf{u}_{\mathbf{t}}\right\} \neq\{0\}}\left\{\left\|\sum_{j=1}^{k_{1}} u_{l_{j}} \mathbf{g}_{\mathbf{i j}}\right\|\right\}\right\},
$$

para cada conjunto possível de normas $\left\{N_{k_{1}}, \ldots, N_{\mathrm{I}}\right\}$ dos cosets $\mathrm{g}_{\mathrm{ij}}$, onde o mínimo $e$ tomado, considerando os elementos do conjunto $\mathcal{U}$ (blocos de bits de entrada), e o máximo na expressão é calculado dentre os líderes de cosets satisfazendo a um mesmo conjunto de normas.

b) A partir dos valores obtidos, construa a Tabela Ordenada de Cosets e Normas constituída de 5 colunas (conjunto de normas $\left\{N_{k_{1}}, \ldots, N_{1}\right\}$; conjunto de $k_{1}$ líderes de cosets; conjunto de blocos de bits de entrada, $\left\{u_{l}\right\}$; $\min \left\{u_{t}\right\} \neq\{0\}\left\{\left\|\sum_{j=1}^{k_{2}} u_{l_{j}} g_{i j}\right\|\right\}$; valor da expressão (7) ) e tantos blocos de linhas, quantas forem os conjuntos possíveis de $k_{1}$ normas da partiçāo (excetuando o conjunto todo nulo). O número de linhas em cada bloco é determinado pelas combinaçôes, dentre os líderes de cosets distintos, para um mesmo conjunto de normas $\left\{N_{k_{1}}, \ldots, N_{1}\right\}$. Os blocos são ordenados de forma decrescente, quanto ao valor da expressão (7) e obtidos através dos mesmos procedimentos.

Para exemplificar, considere $q=2, k_{1}=2$ e a partição $\mathcal{Z}^{2} / \theta \mathcal{Z}^{2}$ conforme a fig.5. Seja o conjunto de normas $\left\{N_{2}, N_{1}\right\}=\{2,2\}$, bloco 2 da tabela ordenada (fig.6), a qual está dividida em 7 blocos de linhas, referentes aos conjuntos $\left\{N_{2}, N_{1}\right\}$. $O$ conjunto $\{2,2\}$ apresenta 4 possibilidades de combinaçōes de líderes de cosets, para esta partiçāo (coluna 2 do bloco 2), pois a mesma possui 2 cosets com norma igual a 2. Como $\mathcal{A}=\mathcal{Z}_{2}$ e $\mathcal{U}=\{\{1,0\},\{0,1\},\{1,1\}\}$ obtem-se a terceira coluna da tabela fazendo as combinaçōes $\left|\sum_{i=1}^{k_{1}} u_{i j} g_{i j}\right|$. A penúltima coluna é obtida da anterior, considerando o valor mínimo da linha; e a última, apresenta o valor máximo (7), dentre os valores da coluna anterior.

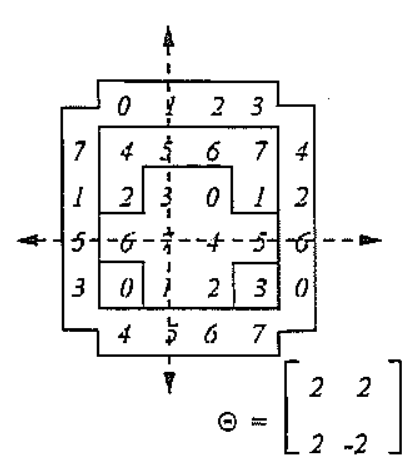

\begin{tabular}{|c|c|c|}
\hline Rótulo & $\begin{array}{c}\text { Líderes de } \\
\text { Coscts }\end{array}$ & Norma \\
\hline 0 & $(1,1)$ & 2 \\
1 & $(0,-1)$ & 1 \\
2 & $(1,-1)$ & 2 \\
3 & $(0,1)$ & 1 \\
4 & $(1,0)$ & 1 \\
$j$ & $(2,0)$ & 4 \\
6 & $(-1,0)$ & 1 \\
7 & $(0,0)$ & 0 \\
\hline
\end{tabular}

FIG. 5: Partiçāo $\mathcal{Z}^{2} / \theta \mathcal{Z}^{2}$ e tabela de líderes e normas(8 cosets).

c) Os blocos referentes aos conjuntos de normas, $\left\{N_{1}, \ldots, N_{k_{1}}\right\}$, que representam os mesmos valores em ordem reversa, são omitidos, pois representam repetiçăo do bloco de linhas dado por $\left\{N_{k_{1}}, \ldots, N_{1}\right\}$, exceto pela ordem de apresentação da terceira coluna.

d) O par de parâmetros, $\left(k_{1}, V\right)$, determina o número de colunas da matriz geradora $\mathrm{G}$, bem como da matriz-norma, GN. As $k_{1}$ primeiras e últimas colunas, se referem aos ramos da treliça que chegam e partem do estado zero(fig.3), sendo estas, as colunas envolvidas na definiçāo do $\Delta_{\text {inf }}$, expressāo (5) Logo, escotha o melhor conjunto de normas para representá-las na matriz-norma GN, de modo que o valor obtido na expressão seja máximo.

As colunas intermediárias de GN, influenciam na maximização do $\Delta_{s u p}$, expressão (6), e devem ser escolhidas a partir dos primeiros blocos de normas listados na tabela ordenada.

Procedendo deste modo, a matriz-norma GN escolhida, determina um subconjunto especial a ser considerado no algoritmo de procura de códigos ótimos.

\section{ALGORITMO GERAL DE PRO- CURA DE CÓDIGOS ÓTIMOS Q-ÁRIOS}

A fig.7 apresenta o fluxograma do algoritmo de procura de códigos ótimos, o qual é uma evolução das versőes apresentadas em [.[2],[3],[4],[5]].

Os detalhes do algoritrono estão apresentados em subseçôes, sendo que, as duas primeiras se referem aos passos do algoritmo de procura que estão inseridos na fase inicial do mesmo, os quais são processados apenas uma vez.

\subsection{Construção da Tabela Ordenada de Co- sets e Normas}

A partir da partição $\Lambda / \Lambda^{\prime}$ e da tabela de líderes de cosets e normas correspondentes (ex. : fig.5), construa a Tabela Ordenada de Cosets e Normas (ex. : fig.6), considerando os conjuntos de normas $\left\{N_{k_{1}}, \ldots, N_{1}\right\}$ (Seção 2). 


\subsection{Escolha da matriz-norma GN}

1) Coloque nas $k_{1}$ primeiras e últimas colunas de GN, o primeiro conjunto de normas da tabela ordenada.

2) Coloque nas colunas intermediárias de GN, o primeiro conjunto de normas, tantas vezes quanto se fizer necessário, e, não sendo possível colocar o conjunto todo (caso o número de colunas intermediárias não seja múltiplo de $k_{1}$ ) retire do mesmo, as menores normas, até a obtenção do número exato de colunas.

\subsection{Cálculo dos valores máximos de $\Delta_{\text {inf }} \mathrm{e}$ $\Delta_{\text {sup }}$ para GN}

A partir da Tabela Ordenada de Cosets e Normas, calculase a expressão (5), para o $\Delta_{i n f}$, considerando o valor máximo da quarta coluna da referida tabela. No cálculo do maior valor para a expressāo (6) $\left(\Delta_{\text {sup }}\right)$, considere os valores móximos da terceira coluna, para cada bloco de bits de entrada, de cada conjunto de normas envolvido.

Por exemplo, seja $G N=\left[\begin{array}{ll|l|ll|ll}4 & 2 & 1 & 1 & 2 & 2\end{array}\right] \quad\left(k_{1}=\right.$ $2, V=4, q=2, \Lambda / \Lambda^{\prime}=\mathcal{Z}^{2} / \theta \mathcal{Z}^{2}$ ). No cálculo da expressão (5), estão envolvidos os dois primeiros blocos de linhas da tabela ordenada. O primeiro, referente às columas $\left\{\left\|g_{22}\right\|\left\|g_{21}\right\|\right\}$ e o segundo, às colunas $\left\{\left\|g_{02}\right\|\left\|g_{01}\right\|\right\}$. Note que, a quarta coluna do primeiro bloco da tabela possui o mesmo valor nas duas linhas, o que não ocorre no segundo bloco, sendo então considerado o valor máximo, que $e ́$ 2. Obtem-se então :

$$
\Delta_{\text {inf }}=2+2=4 .
$$

Para calcular o valor máximo do $\Delta_{s u p}$, estão envolvidos os três primeiros blocos, correspondendo respectivamente, às colunas $\left\{\left\|\mathrm{g}_{22}\right\|\left\|\mathrm{g}_{21}\right\|\right\},\left\{\left\|\mathrm{g}_{02}\right\|\left\|\mathrm{g}_{01}\right\|\right\}$ e $\left\{\left\|\mathbf{g}_{12}\right\|\left\|\mathbf{g}_{11}\right\|\right\}$. Em cada um desses blocos, considere 0 maior valor para cada bloco de bits de entrada Assim

$$
\begin{aligned}
\Delta_{\text {sup }} & =\min \{(2+1+4),(2+1+2),(4+4+2)\} \\
& =\min \{7,5,10\}=5 .
\end{aligned}
$$

\subsection{Procura dos Melhores Códigos em um Subconjunto Especial}

O fluxograma apresentado na fig.8 descreve esta fase do algoritmo de procura de códigos ótimos.

Uma matriz-norma GN, que represente um subconjunto especial de matrizes geradoras $\mathrm{G}$, onde todos os códigos são, ou catastróficos ou não impliquem a escolha de todos os cosets da partiçäo, é prontamente descartada, sem que o algoritmo de determinação do $d_{f r e e}$ seja acionado uma única vez.

\subsection{Nova Escolha de GN}

Nesta fase, apenas uma das etapas abaixo é realizada, conforme a existência de novas possibilidades de escolha, sendo observada a ordem de precedência.

\begin{tabular}{|c|c|c|c|c|c|c|c|}
\hline$k_{1}=2$ & 8 cosels & & $A=C$ & $G F(2)$ & & & \\
\hline$\left\{N_{1}, N_{2}\right\}$ & $\begin{array}{l}\text { Lideres dos } \\
\text { Cosels }\end{array}$ & $\{1,0\}$, & $\{0,1\}$ &,$\{1,1\}$ & $\min \{\}$. & Eq. (7) & \\
\hline$\{4,2\}$ & $(0,2),(1,1)$ & 4 & , 2 & $\cdot 2$ & 2 & 2 & \\
\hline & $(0,2),(1-, 1)$ & &,$\quad 2$ &, 2 & 2 & & \\
\hline$\{2,2\}$ & $(1,1),(1,1)$ & 2 &,$\quad 2$ & $=0$ & 0 & & \\
\hline & $(1,1),(1,-1)$ & 2 &,$\quad 2$ & $\sqrt{2}$ & 2 & 7 & - \\
\hline & $(1,-1),(1,1)$ & 2 & 2 & , 4 & 2 & 2 & $B L O C O$ \\
\hline & $(1,-1),(1,-1)$ & 2 & .2 &, 0 & 0 & & \\
\hline$\{1,1\}$ & $(1,0),(1,0)$ & 1 &, 1 &, 4 & I & & \\
\hline & $(1,0),(0,1)$ & 1 & , 1 &, 2 & $\perp$ & & \\
\hline & $(1,0),(-1,0)$ & 1 & , I &, 0 & 0 & & \\
\hline & $(1,0),(0,-1)$ & 1 & , I &, 2 & 1 & & \\
\hline & $(0,1),(1,0)$ & 1 & . 1 & , 2 & 1 & & \\
\hline & $(0,1),(0,1)$ & 1 & , 1 &,$\quad 2$ & 1 & & \\
\hline & $(0,1),(-1,0)$ & 1 &,$\quad 1$ &, 2 & 1 & & \\
\hline & $(0,1),(0,-1)$ & 1 & , 1 &, 0 & 0 & 1 & \\
\hline & $(-1,0),(1,0)$ & 1 &,$\quad 1$ &, 0 & 0 & 1 & \\
\hline & $(-1,0),(0,1)$ & 1 &,$\quad 1$ &, 2 & 1 & & \\
\hline & $(-1,0),(-1,0)$ & 1 & , I &, 4 & 1 & & \\
\hline & $(-1,0),(0,-1)$ & $i$ &,$\quad I$ &, 2 & 1 & & \\
\hline & $(0,-1),(1,0)$ & j & , I & , 2 & 1 & & \\
\hline & $(0,-1),(0,1)$ & 1 & , I &, 4 & 1 & & \\
\hline & $(0,-1),(-1,0)$ & 1 &, 1 &, 2 & 1 & & \\
\hline & $(0,-1) ;(0,-1)$ & 1 & +1 &, 4 & 1 & & \\
\hline$\{4,1\}$ & $(0,2),(0,1)$ & 4 &, 1 & . 1 & 1 & & \\
\hline & $(0,2),(1,0)$ & 4 &, 1 & .1 & 1 & 1 & \\
\hline & $(0,2),(0,-1)$ & 4 &,$\quad 1$ &, 1 & 1 & 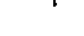 & \\
\hline & $(0,2),(-1,0)$ & 4 &,$\quad 1$ &, 1 & 1 & & \\
\hline$\{2,1\}$ & $(1,1),(1,0)$ & 2 &, 1 &, 1 & 1 & & \\
\hline & $(1,1),(0,1)$ & 2 &, 1 &, 1 & 1 & & \\
\hline & $(1,1),(-1,0)$ & 2 &,$\quad I$ &, 1 & I & & \\
\hline & $(1,1),(0,-1)$ & 2 & , I &,$\quad t$ & 1 & 1 & \\
\hline & $(1,-1),(1,0)$ & 2 &,$\quad 1$ & , 1 & I & & \\
\hline & $(1,-1),(0,1)$ & 2 &,$\quad 1$ &, 1 & 1 & & \\
\hline & $(1,-1),(-1,0)$ & 2 &,$\quad 1$ & , 1 & 1 & & \\
\hline & $(1,-1),(0,-1)$ & 2 & $=1$ &, 1 & 1 & & \\
\hline$\{0,1\}$ & $(0,0),(0,1)$ & 0 & , 1 & , 0 & 0 & & \\
\hline & $(0,0),(1,0)$ & 0 &,$\quad 1$ &, 0 & 0 & 0 & \\
\hline & $(0,0),(0,-1)$ & 0 &,$\quad 1$ &, 0 & 0 & & \\
\hline & $(0,0),(-1,0)$ & 0 &, 1 &, 0 & 0 & & \\
\hline$\{4-4\}$ & $(0,2),(0,2)$ & 4 &, 4 & $=0$ & 0 & 0 & \\
\hline
\end{tabular}

a) Se for possível, substitua as colunas intermediárias da GN anterior, por nova escolha do conjunto de normas, sempre
FIG. 6: Tabela Ordenada de Cosets e Normas.

observando-se a ordem subsequente dos conjuntos segundo a Tabela Ordenada de Cosets e Normas, utilizanco inclusive a ordem reversa dos conjuntos $\left\{N_{1}, \ldots, N_{k_{1}}\right\}$. Escolhida nova GN, retorne à seçāo 3.3. Caso contrário, etapa (b) a seguir.

b) Se for possível, troque o conjunto de normas que compõem as. $k_{1}$ últimas colunas de $\mathrm{GN}$, utilizando o próximo bloco de normas da tabela ordenada Para as colunas intermediarias, tome a primeira combinação utilizada e continue o algoritmo de procura (seção 3.3). Caso contrório, etapa(c).

c) Troque o conjumto de normas que compōe o primeiro bloco de $k_{1}$ colunas de GN, retome a primeira combinação de normas utilizada para as demais colunas e continue (seção 3.3). 


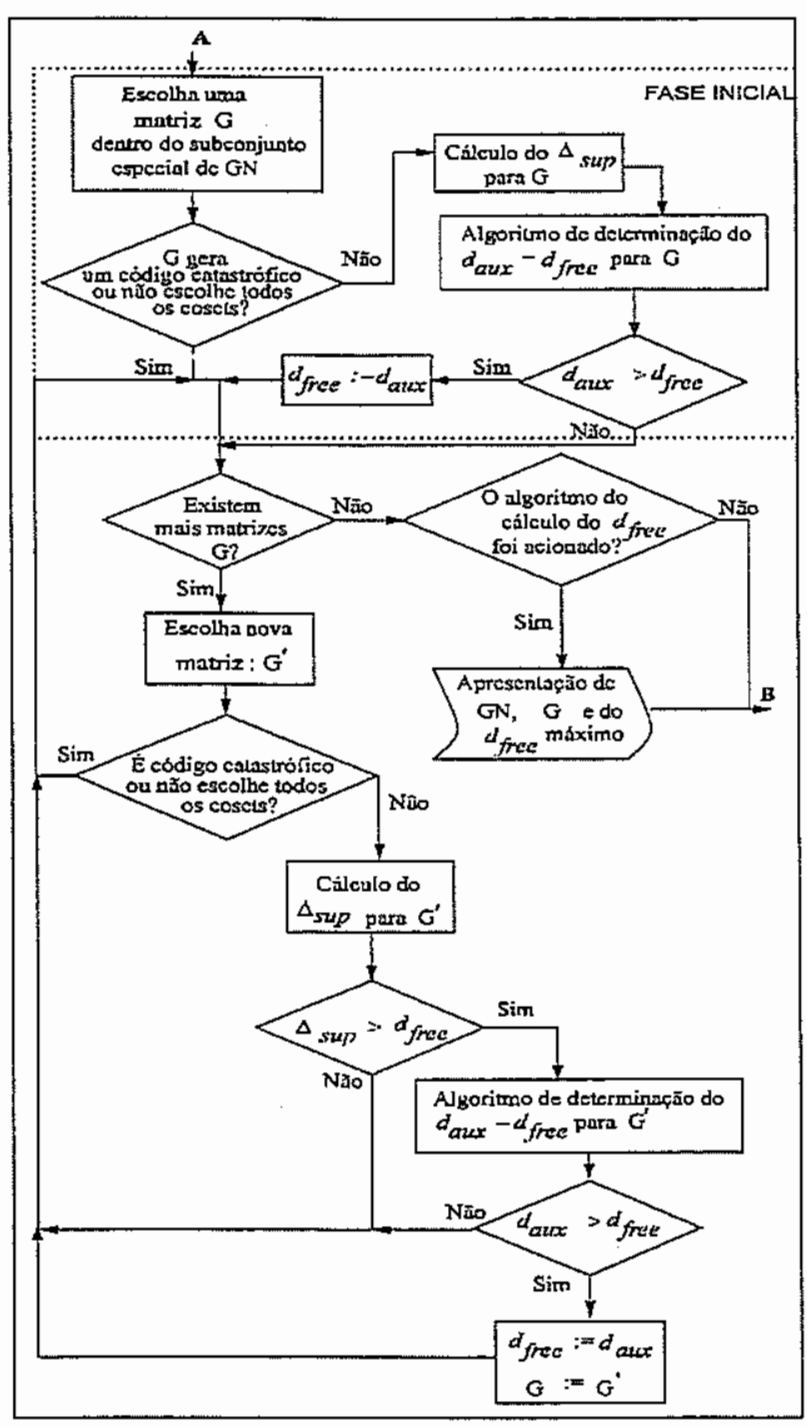

FIg. 7: Fluxograma do Algoritmo de Procura de Códigos Ótmos Q-ários.

\subsection{Apresentação do Código Ótimo (final do algoritmo)}

A determinação do código ótimo e do $d_{\text {free }}$ máximo para um dado conjunto de parâmetros, $\left(k_{1}, V, q\right)$ e partição de reticulados é obtida ao se esgotar as possibilidades de nova escolha de GN, i.e., o algoritmo sempre determina um código otimo. Ressalta-se que a procura de códigos ótimos realizada é exaustiva quanto à escolha das matrizes-norma GN e das matrizes geradoras $\mathrm{G}$, ou seja, procura-se em todos os subconjuntos especiais possíveis, utilizando entretanto, o cálculo do $d_{\text {free }}$, para uma fração mínima de matrizes geradoras $\mathrm{G}$ [[3],[4],[5],[6]].

\section{EXEMPLOS NUMÉRICOS}

\subsection{Códigos Treliça Binários :}

$$
k_{1}=2 ; V=4 ; q=2 ; \Lambda / \Lambda^{\prime}=\mathcal{Z}^{2} / \theta \mathcal{Z}^{2}(8 \text { cosets })
$$

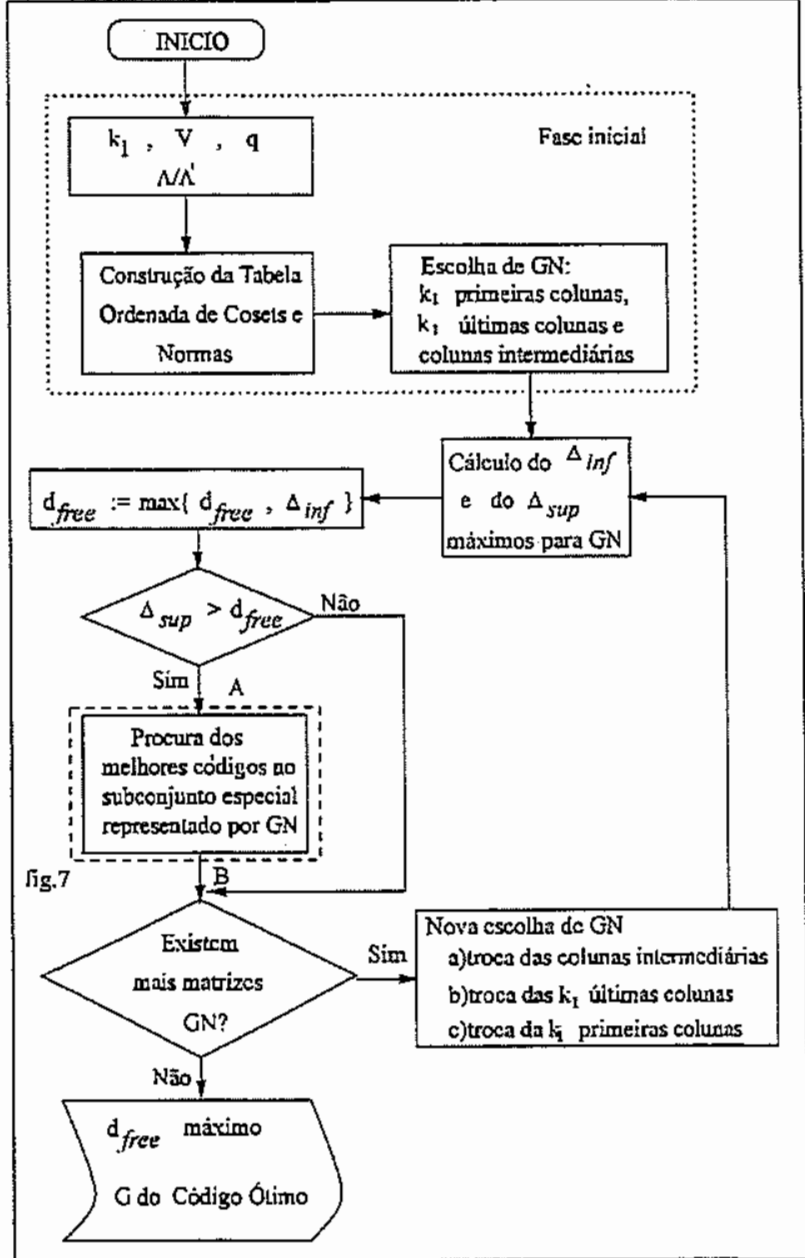

Fig. 8: Fluxograma da Procura dos Melhores Codigos em um Subconjunto Especial

Este exemplo aparece, resumidamente, em [4].

A partir da partiçāo e da tabela de líderes de cosets e normas (fig.5), construiu-se, segundo a seção 2.2, a Tabela Ordenada de Cosets e Normas, apresentada na fig. 6 .

A matriz-norma possui $k_{1}+V$ colunas, logo:

$G N=\left[\left\|\mathrm{g}_{22}\right\|\left\|\mathrm{g}_{21}\right\|\left|\left\|\mathrm{g}_{12}\right\|\left\|\mathrm{g}_{11}\right\|\right|\left\|\mathrm{g}_{02}\right\|\left\|\mathrm{g}_{01}\right\|\right]$

A primeira matriz-norma GN determinada pelo algoritmo apresentado na seção anterior, é :

$$
G N=\left[\begin{array}{llllll|ll}
4 & 2 & 4 & 2 & 4 & 4
\end{array}\right],
$$

cujo $\Delta_{\text {inf }}=4$ e $\Delta_{\text {sup }}=6$.

Porém qualquer combinação de colunas das matrizes geradoras $G$ do subconjunto de GN, produz um coset de norma par. Logo as matrizes geradoras determinam códigos que nāo utilizam todos os cosets da partição considerada.

Desta maneira, a matriz GN considerada é descartada. O mesmo ocorre, neste exemplo, para qualquer matriz GN que não possua, pelo menos, uma coluna com norma ímpar. Assim as matrizes-norma são geradas na seguinte ordem:

1) $G N=\left[\begin{array}{ll|ll|ll}4 & 2 & 1 & 1 & 4 & 2\end{array}\right], \Delta_{i n f}=2+2=4$ e $\Delta_{\text {sup }}=\min \{5,9,8\}$. Acionado o algoritmo do cálculo do 
$d_{\text {free }}$ para este subconjuto especial, obtem-se, pelo menos um código com $d_{a u x}=5$. Faz-se $d_{\text {free }}=5$, guardando também a matriz geradora correspondente, $\mathrm{G}$.

2) Mantidas as colunas 1, 2,5 e 6 e trocando as colunas 3 e 4 , sequencialmente, a partir dos melhores valores da tabela ordenada (fig.6), que são $(4,1),(1,4),(2,1),(1,2),(0,1)$ e $(1,0)$, tem-se matrizes $\mathrm{GN}$ com $\Delta_{\text {sup }}=5$, exceto a última que tem $\Delta_{\text {sup }}=4$. Como o $d_{\text {free }}$ desses subconjuntos especiais não podem superar $o d_{a u x}$ já obtido, descarte-se prontamente os subconjuntos, sem que o algoritmo do cálculo do $d_{\text {free }}$ seja acionado.

3) $G N=\left[\begin{array}{ll|ll|ll}4 & 2 & 1 & 1 & 2 & 4\end{array}\right], \Delta_{\text {inf }}=4$ e $\Delta_{\text {sup }}=$ $\min \{7,7,8\}$. Inspecionado o subconjuto especial de GN, obtem-se, no máximo, $d_{a u x}=6$. Faz-se $d_{f r e e}=6$ e guardase esta nova matriz geradora.

4) Mantidas as colunas $1,2,5$ e 6 e trocando as colunas 3 e 4 , sequencialmente, pelos valores $(4,1),(1,4),(2,1)$, $(1,2),(1,0)$ e $(0,1)$, as matrizes GN resultantes possuem todas, $\Delta_{\text {sup }}=5$, o qual é menor que o $d_{a u x}$. Portanto são prontamente descartadas.

5) As matrizes GN : [ $\left.\begin{array}{ll|l|l|ll}4 & 2 & 1 & 1 & 2 & 2\end{array}\right]$ e $\left[\begin{array}{lllll|lll}4 & 2 & 4 & 1 & 2 & 2\end{array}\right]$ possuem $\Delta_{\text {sup }}=5$ e portanto, prontamente descartadas.

6) $G N=\left[\begin{array}{ll|ll|ll}4 & 2 & 1 & 4 & 2 & 2\end{array}\right], \quad \Delta_{i n f}=4 \quad \mathrm{e}$ $\Delta_{\text {sup }}=\min \{8,7,7\}$. Dentre as matrizes geradoras do subconjuto especial de GN, obtem-se, no máximo, $d_{a u x}=5 \mathrm{e}$ portanto descarta-se GN, visto que $d_{\text {free }}=6$ já foi obtido.

7) As matrizes GN : [ $\left.\begin{array}{ll|l|l|llll}4 & 2 & 2 & \text { I } & 2 & 2\end{array}\right] \mathrm{e}$ $\left[\begin{array}{lllll|ll}4 & 2 & 1 & 2 & 2 & 2\end{array}\right]$ implicam em $\Delta_{\text {sup }}=5$ e 6 , e portanto são prontamente descartadas.

8) $G N=\left[\begin{array}{ll|ll|lll}2 & 4 & 1 & 1 & 4 & 2\end{array}\right], \quad \Delta_{i n f}=4 \quad \mathrm{e}$ $\Delta_{\text {sup }}=\min \{7,7,8\}$. Dentre as matrizes geradoras do subconjuto especial de GN, obtem-se, no máximo, $d_{a u x}=6$,

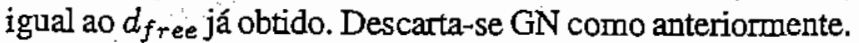

9) $G N=\left[\begin{array}{ll|ll|ll}2 & 4 & 4 & 1 & 2 & 2\end{array}\right], \quad \Delta_{\text {inf }}=4 \mathrm{e}$ $\Delta_{\text {sup }}=\min \{8,7,7\}$. Obtem-se, no máximo, $d_{\text {free }}=5$. Descartada, como anteriores.

As demais matrizes [ $\left[\begin{array}{ll|l|l|ll}2 & 4 & 2 & 1 & 2 & 2\end{array}\right]$, [ $\left[\begin{array}{ll|ll|ll}2 & 4 & 4 & 4 & 2 & 2\end{array}\right], \ldots,\left[\begin{array}{ll|l|l|ll}2 & 4 & 4 & 2 & 1 & 1\end{array}\right], \ldots$, possuem $\Delta_{\text {sup }}$ menores que o $d_{\text {free }}$ já obtido e portanto são todas prontamente descartadas.

Com isto, esgota-se todos os possíveis subconjuntos especiais para este esquema de códigos treliça de parâmetros $\left(k_{1}, V, q\right)=(2,4,2)$. O código convolucional binário ótimo foi obtido no subconjunto especial representado pela matriznorma $G N=\left[\begin{array}{ll|ll|ll}4 & 2 & 1 & 1 & 2 & 4\end{array}\right]$ e cuja matriz geradora é

$$
G=\left(\begin{array}{llllll}
0 & 1 & 1 & 0 & 1 & 0 \\
2 & 1 & 0 & 1 & 1 & 2
\end{array}\right)
$$

Observe que, para códigos cujo alfabeto é $\mathcal{A}=G F(2)$ e a partição é $\mathcal{Z}^{2} / \theta Z^{2}$ (8 cosets), de um universo de 4095 possíveis formas para a matriz-norma GN, inspecionamos,

\begin{tabular}{|c|c|c|c|c|c|c|c|c|}
\hline Ronulo & $\begin{array}{l}\text { Lideres de } \\
\text { Cosets }\end{array}$ & Norma & Roculo & $\begin{array}{c}\text { Lideres ie } \\
\text { Cosets }\end{array}$ & Noma & Rotulo & $\begin{array}{c}\text { Lideres } \\
\text { Cosets }\end{array}$ & Norms \\
\hline 0 & $(0,0)$ & 0 & 9 & $(2,2)$ & 2 & 18 & $(-2,0)$ & 2 \\
\hline 1 & $(1,0)$ & 1 & 10 & $(1,2)$ & $\hat{3}$ & 19 & $(-1,0)$ & $i$ \\
\hline 2 & $(1,1)$ & 1 & II & $(0,2)$ & 2 & 20 & $(3,3)$ & 9 \\
\hline 3 & $(0,1)$ & 1 & 12 & $(0,-2)$ & 2 & 21 & $(-2,-2)$ & 2 \\
\hline 4 & $(2,0)$ & 2 & 13 & $(-1,1)$ & 3 & 22 & $(-1,-2)$ & 3 \\
\hline 5 & $(-1,2)$ & 7 & 14 & $(1,-2)$ & 7 & 23 & $(-3,-2)$ & 7 \\
\hline 6 & $(0,-1)$ & 1 & 15 & $(0,3)$ & 9 & 24 & $(-2,-1)$ & 3 \\
\hline 7 & $(2,1)$ & 3 & 16 & $(2,3)$ & 7 & 25 & $(-1,-1)$ & 1 \\
\hline 8 & $(2,-1)$ & 3 & 17 & $(1,3)$ & 7 & 26 & $(3.2)$ & 7 \\
\hline
\end{tabular}

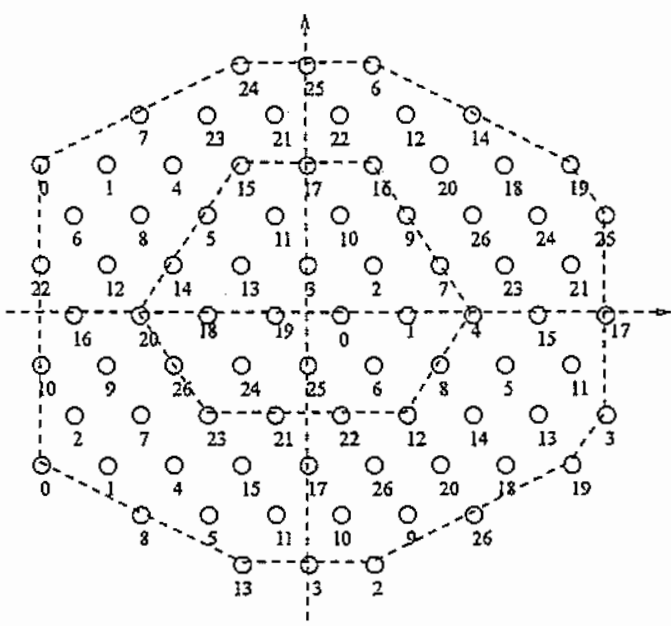

FIG. 9: Partição $\mathcal{A}_{2} / 9 \mathcal{A}_{2}$ e tabela de líderes de cosets e normas (27 cosets).

com efetivo cálculo do $d_{\text {free }}$, os subconjuntos especiais associados à apenas cinco delas. Os 4090 subconjuntos restantes foram prontamente descartados sem o efetivo cálculo do $d_{\text {free }}$.

\subsection{Códigos Treliça Temários :}

$$
k_{1}=1 ; V=2 ; q=3 ; \Lambda / \Lambda^{\prime}=\mathcal{A}_{2} / 9 \mathcal{A}^{2}(27 \text { cosets })
$$

A partir da partição e da tabela de líderes de cosets e normas (fig.9), construiu-se, segundo a seção 2.2 , a Tabela Ordenada de Cosets e Normas, apresentada na fig.10.

As matrizes norma $\mathrm{GN}$, aqui da forma

$$
G N=\left[\begin{array}{lll}
g_{31} & g_{21} & g_{01}
\end{array}\right],
$$

são escolhidas segundo o algoritmo de procura de códigos da seção III, na seguinte ordem :

$$
\text { 1) } G N=\left[\begin{array}{lll}
9 & 9 & 9
\end{array}\right], \Delta_{i n f}=9+9=18 \mathrm{e}
$$

$\Delta_{\text {sup }}=\min \{27,27\}$. Esta matriz GN é descartada sem o acionamento do cálculo do $d_{f r e e}$, juntamente com todas as matrizes geradoras $\mathrm{G}$ associadas ao subconjunto especial, pois não possibilitam a escolha de todos os cosets da partição na saída do codificador.

2) $G N=\left[\begin{array}{lll}9 & 3 & 9\end{array}\right], \quad \Delta_{\text {inf }}=18$ e $\Delta_{\text {sup }}=$ $\min \{21,21\}$. Descartada pelo mesmo motivo da anterior.

3) $G N=\left[\begin{array}{lll}9 & 2 & 9\end{array}\right], \quad \Delta_{i n f}=18$ e $\Delta_{\text {sup }}=$ $\min \{20,25\}$. Descartada pelo mesmo motivo da anterior. 


\begin{tabular}{|c|c|c|c|c|}
\hline$k_{1}=1$ & 27 cosets ; & $\mathrm{A}=\mathrm{GF}(3)$ & & \\
\hline Norma & Lider do Coset & $\|g i j \mid\| 2 g i j \|$, & $\min \{\}$. & Eq. (7) \\
\hline$\{9\}$ & $\begin{array}{l}(3,3) \\
(3,3)\end{array}$ & $\begin{array}{l}9,9 \\
9,9\end{array}$ & $\begin{array}{l}9 \\
9\end{array}$ & 9 \\
\hline$\{3\}$ & $\begin{array}{l}(2,1) \\
(2,-1) \\
(1,2) \\
(-1,1) \\
(-1,-2) \\
(-2,-1)\end{array}$ & $\begin{array}{ll}3, & 3 \\
3, & 3 \\
3, & 3 \\
3, & 3 \\
3, & 3 \\
3, & 3\end{array}$ & $\begin{array}{l}3 \\
3 \\
3 \\
3 \\
3 \\
3\end{array}$ & 3 \\
\hline$\{2\}$ & $\begin{array}{l}(2,0) \\
(2,2) \\
(0,2) \\
(0,-2) \\
(-2,0) \\
(-2,-2)\end{array}$ & $\begin{array}{l}2,7 \\
2,7 \\
2,7 \\
2,7 \\
2,7 \\
2,7\end{array}$ & $\begin{array}{l}2 \\
2 \\
2 \\
2 \\
2 \\
2\end{array}$ & 2 \\
\hline$\{7\}$ & $\begin{array}{l}(-1,2) \\
(1,-2) \\
(2,3) \\
(1,3) \\
(-3,-2) \\
(3,2)\end{array}$ & $\begin{array}{ll}7, & 1 \\
7, & 1 \\
7, & 1 \\
7, & 1 \\
7, & 1 \\
7, & 1\end{array}$ & $\begin{array}{l}1 \\
1 \\
1 \\
1 \\
1 \\
1\end{array}$ & 1 \\
\hline$\{1\}$ & $\begin{array}{l}(1,0) \\
(1,1) \\
(0,1) \\
(0,-1) \\
(-1,0) \\
(-1,-1)\end{array}$ & $\begin{array}{ll}1, & 2 \\
1, & 2 \\
1, & 2 \\
1, & 2 \\
1, & 2 \\
1, & 2\end{array}$ & $\begin{array}{l}1 \\
1 \\
1 \\
1 \\
1 \\
1\end{array}$ & 1 \\
\hline
\end{tabular}

FIG. 10: Tabela Ordenada de Cosets e Normas.

4) $G N=\left[\begin{array}{lll}9 & 1 & 9\end{array}\right], \quad \Delta_{i n f}=18$ e $\Delta_{\text {sup }}=$ $\min \{19,20\}$. Descartada pelo mesmo motivo da anterior.

5) As matrizes-norma $G N=\left[\begin{array}{lll}9 & 9 & 3\end{array}\right] \mathrm{e}$ $G N=\left[\begin{array}{lll}9 & 3 & 3\end{array}\right]$, implicam em $\Delta_{\text {inf }}=12$, e $\Delta_{\text {sup }}=21$ e 18 , respectivamente. Como também não escolhem todos os cosets, são descartadas.

6) $G N=\left[\begin{array}{lll}9 & 2 & 3\end{array}\right], \Delta_{i n f}=12$ e $\Delta_{\text {sup }}=$ $\min \{14,19\}$. O subconjunto especial é inspecionado e um código com $d_{a u x}=14$ é obtido. Faz-se $d_{\text {free }}=14$ e guardase G.

7) As matrizes-norma $G N=\left[\begin{array}{lll}9 & 7 & 3\end{array}\right]$ e $G N=\left[\begin{array}{lll}9 & 1 & 3\end{array}\right]$ possuem $\Delta_{\text {sup }}=13$. Logo são prontamente descartadas, pois $\Delta_{\text {sup }}<d_{\text {free já obtido. }}$

8) $G N=\left[\begin{array}{lll}9 & 9 & 2\end{array}\right]$, não escolhe todos os cosets da partição, portanto é descartada.

9) $G N=\left[\begin{array}{lll}9 & 3 & 2\end{array}\right]$ e $G N=\left[\begin{array}{lll}9 & 2 & 2\end{array}\right]$. Descartadas prontamente, pois $\Delta_{\text {sup }} \leq d_{\text {free }}$ obtido anteriormente.

10) $G N=\left[\begin{array}{lll}9 & 7 & 2\end{array}\right], \quad \Delta_{\text {inf }}=12$ e $\Delta_{\text {sup }}=$ $\min \{18,17\}$. Investigado o subconjunto das matrizes $G$ obtem-se $d_{a u x}=16$, o qual é máximo até o momento. Faz-se $d_{\text {free }}=16$ e guarda-se $\mathrm{G}$.
11) As matrizes $G N=\left[\begin{array}{lll}3 & 9 & 9\end{array}\right],\left[\begin{array}{lll}3 & 3 & 9\end{array}\right]$, $\left[\begin{array}{lll}3 & 2 & 9\end{array}\right],\left[\begin{array}{lll}3 & 7 & 9\end{array}\right],\left[\begin{array}{lll}3 & 1 & 9\end{array}\right],\left[\begin{array}{lll}3 & 9 & 3\end{array}\right]$, bem como todas as demais são descartadas prontamente pois, ou não escolhem todos os cosets, ou possuem $\Delta_{\text {sup }} \leq d_{\text {aux }}$.

Assim são esgotadas todas as possibilidades para as matrizes-norma $G N$ e matrizes geradoras $G$ de todos os subconjuntos especiais possíveis para este esquema. A procura para códigos treliça ternários, com parâmetros $\left(k_{1}, V\right)=$ $(1,2)$ e 27 cosets na partição, é concluída com a apresentação de um código convolucional ótimo, gerado por $\mathrm{G}$, com $d_{\text {free }}=16$,

$$
G=\left(\begin{array}{lll}
0 & 1 & 0 \\
3 & 3 & 2
\end{array}\right) \quad \text { e } \quad G N=\left[\begin{array}{lll}
9 & 7 & 2
\end{array}\right] .
$$

Salienta-se que de um universo de 215 formas possíveis para a matriz norma GN, 213 são prontamente descartadas, sem que haja o efetivo cálculo do $d_{\text {free }}$ para todas as matrizes geradoras contidas nestes 213 subconjuntos especiais.

\section{CONCLUSÃO}

O algoritmo geral proposto, determina códigos convolucionais ótimos, envolvidos na estrutura dos códigos treliça [1] baseados em cosets e partiçōes de reticulados. O mesmo incorpora os procedimentos propostos em [[2],[4]] e generaliza a procura, quanto ao alfabeto de entrada, podendo ser um anel com q elementos [5].

Com relação as suas versões anteriores [.[2],[3],[4],[5]] a apresentação do algoritmo, aqui realizada (seção 3), é bastante precisa e detalhada, tendo como características fundamentais, a construção da Tabela Ordenada de Cosets e Normas (seção 2) e sua utilização, tanto na escolha eficaz da matriz-norma GN (seção 3.2 e 3.5), quanto no cálculo dos valores máximos para $\Delta_{\text {inf }}$ e $\Delta_{\text {sup }}$ para um subconjunto especial (seção 3.3). Estas características salientam, ainda mais, o caráter exaustivo de procura de códigos ótimos, visto que, se esgotam todas as possibilidades para as matrizes-norma GN e matrizes geradoras $\mathrm{G}$ de todos os subconjuntos especiais para um dado código treliça.

A procura de melhores códigos em um subconjunto especial (seção 3.4) é apresentada, através de um fluxograma (fig.8), destacado do algoritmo geral, o que permite uma completa visão desta fase, sem o prejuízo da visão total do algoritmo proposto.

Ressalta-se também que, dentro dos subconjuntos especiais, o cálculo do $d_{\text {free }}$ é acionado para uma fração mínima de matrizes geradoras, neles contidas. Este fato pôde ser observado nos exemplos apresentados neste artigo para códigos treliça binários e ternários (seção 4.1 e 4.2), bem como no exemplo para códigos treliça quaternário, apresentado em .[5] em outros exemplos em .[6].

\section{AGRADECIMENTOS}

Este trabalho foi parcialmente suportado por uma bolsa PICD/CAPES para a professora E.M. Rosa. 


\section{REFERÊNCIAS}

[1] Calderbank,A.R. e Sloane,N.J.A. New Trellis Codes based on Lattices e Cosets. IEEE Trans. Inform.Theory, Vol.IT-33, pp.177-195, Mar.1987.

[2] Rosa,EM. e Borelli,W.C. Procedimentos Simples para a procura de Códigos Ótimos, através de Matrizes Geradoras representadas por Cosets. XIV Simpósio Brasileiro de Telecomunicações, Vol.2, pp.655-660, Curitiba, PR, Brasil, Julho 1996.

[3] Rosa,E.M., Borelli,W.C. e Farrell,P.G. Special Subsets e Optimum Codes based on Lattices e Cosets. Intenational Symposium of the Informations Theory (ISIT'97), p.521, Ulm, Germany, June 29 - July 4,1997.

[4] Rosa,EM., Borelli,W.C. e Farrell,P.G. Novos Procedimentos para a Procura de Códigos Ótimo Binánios baseados em Partiçôes de reticulados. XV Simpósio Brasileiro de Telecomunicaçōes, pp.479-483, Recife, PE, Brasil, Setembro 1997.

[5] Rosa,EM., Borelli,W.C. e Farrell,P.G. A Formalized Optimum Code Search for q-ary Trellis Codes. IEEE Global Telecommunications Conference (GLOBECOM'97), S26p5, ISBN CD-ROOM : 0-7803-4201-1,Phoenix, Arizona, USA, Nov.1997.

[6] Rosa,E.M. Ph.D. Thesis (em preparação) - DT/FEEC/ Unicamp - Campinas - SP - Brasil.

Emília M. Rosa : Professora da Faculdade de Ciências da UNESP/Bauru desde 1990; atualmente é Professora MS-2 do Departamento de Matemática da FC/UNESP. Graduou-se em Matemática, no ano de 1986 pela UNESP, Rio Claro-SP; obteve título de Mestre em Matemática em 1990 pelo IMECC $U$ NICAMP e; atualmente é aluna regular do Curso de Doutoramento em Eng. Elétrica - FEEC/UNICAMP, Campinas-SP.Áreas de pesquisa de atual interesse : Teoria de Informação e codificação : Estruturas algébricas e geométricas para codificaçāo e modulação combinadas (TCM e BCM); Códigos de bloco e convolucionais para transmissão de dados clássica e de multi-usuários; Teoria dos Números.

Walter C. Borelli : Professor da Faculdade de Engenharia Elétrica da UNICAMP desde 1973; atualmente é Professor MS-5 do Departamento de Telemática da FEEC/UNICAMP. Obteve o título de Engenheiro Elétrico em 1972 pela EESC-USP, São Carlos-SP; de Mestre em Engenharia Elétrica em 1975 pela FEC/UNICAMP e de $\mathrm{Ph} D$. em Eng. Eletronica e de Comunicaçōes em 1983, pela Universidade de Kent, Canterbury, Inglaterra. Áreas de pesquisa de anual interesse : a)Teoria de informaçāo e codificação : Estruturas algébricas e geométricas para codificação e modulação combinadas (TCM e BCM); Códigos de bloco e convolucionais para transmissão de dados clássica e de multiusuários; Análise e síntese de circuítos digitais para codificadores e decodificadores de sistemas para correção de erros. b)Especificação, simulação e validação de sistemas complexos : Protocolos para redes ATM; BISDN, Multimídia e Redes sem fio; Eng. de Software; Manufatura; Banco de dados e etc.
Patrick G. Farrell : Former Head of Communications Research Group / Electrical Engineering Division / Faculty of Science and Engineering / The University of ManchesterUK Nowadays owns a part-time Chair at the Communications Research Centre / Faculty of Applied Sciences / University of Lancaster-UK. Concerned with all aspects of digital communication systems theory and pratice. These include error control coding for all types of communication and information storage systems, multifunctional coding, multiple access techniques applied to personal and mobile communication and broadband networks, security and cryptographic protocols, performance analysis and simulation of communication systems, and autonomous systems. 